\title{
Proposal of a new equation for estimating resting energy expenditure of acute kidney injury patients on dialysis: a machine learning approach
}

\author{
Daniela Ponce* ${ }^{*}$, Cassiana Regina de Goes and Luis Gustavo Modelli de Andrade
}

\begin{abstract}
Background: The objective of this study was to develop a new predictive equation of resting energy expenditure (REE) for acute kidney injury patients (AKI) on dialysis.

Materials and methods: A cross-sectional descriptive study was carried out of 114 AKI patients, consecutively selected, on dialysis and mechanical ventilation, aged between 19 and 95 years. For construction of the predictive model, $80 \%$ of cases were randomly separated to training and $20 \%$ of unused cases to validation. Several machine learning models were tested in the training data: linear regression with stepwise, rpart, support vector machine with radial kernel, generalised boosting machine and random forest. The models were selected by ten-fold cross-validation and the performances evaluated by the root mean square error.

Results: There were 364 indirect calorimetry measurements in 114 patients, mean age of $60.65 \pm 16.9$ years and $68.4 \%$ were males. The average REE was $2081 \pm 645 \mathrm{kcal}$. REE was positively correlated with C-reactive protein, minute volume (MV), expiratory positive airway pressure, serum urea, body mass index and inversely with age. The principal variables included in the selected model were age, body mass index, use of vasopressors, expiratory positive airway pressure, MV, C-reactive protein, temperature and serum urea. The final r-value in the validation set was 0.69.
\end{abstract}

Conclusion: We propose a new predictive equation for estimating the REE of AKI patients on dialysis that uses a nonlinear approach with better performance than actual models.

Keywords: Energy metabolism, Resting energy expenditure, Machine learning, Acute kidney injury, Sepsis, Dialysis

\section{Introduction}

Acute kidney injury (AKI) occurs in approximately $3-15 \%$ of hospitalised patients and can affect $30-50 \%$ of patients admitted to intensive care units (ICU). It is associated with extremely high mortality rates, ranging from 20 to $50 \%$ [1]. Previous observational studies reported that malnourished and hospitalised AKI patients have higher rates of morbidity and mortality

\footnotetext{
*Correspondence: daniela.ponce@unesp.br

Department of Internal Medicine - UNESP, Univ Estadual Paulista, Rubião

Jr, s/n - Botucatu/SP18.618-970, São Paulo, Brazil
}

than well-nourished patients [2, 3] and an association between cumulative caloric deficits and poor outcome in ICU patients $[4,5]$. Accurate determination of energy needs is obviously important in critically ill patients as both over and underfeeding may be associated with complications and undesired consequences [6].

Determining energy requirements in critically ill patients via indirect calorimetry (IC) has long been considered the gold standard [7]. Limitations for using IC include time constraints, equipment availability, staffing and cost. Therefore, many predictive equations exist for predicting resting energy expenditure (REE), but 
the accuracy of these equations for estimating caloric requirements of critically ill patients is unclear [7-13]. Goes et al. evaluated if nine different standard predictive equations for energy expenditure could accurately reflect the energy requirements of critically ill, mechanically ventilated AKI patients [14]. There was low precision and poor agreement between measured and predicted REE by the Harris-Benedict (HB), Mifflin, Ireton-Jones, Penn State, American College of Chest Physicians and Faisy equations. The $\mathrm{HB}$, without using the injury factor, was the least precise (18\% precision). Modified Penn State equation had the best precision, although the precision rate was only $41 \%$. In conclusion, none of these equations accurately estimated measured REE in severe AKI patients on dialysis and most of them underestimated energy needs [14]. Recently more sophisticated models using machine learning were applied in clinical practices resulting in better predictive models [15]. These models can be applied to build predictive equations aiming to capture the non-linearity between variables, resulting in better accuracy. These new models have also been applied to better predict resting energy expenditure (REE) [16].

\section{$\mathrm{n}=114$ AKI
Dialysis /Mechanical Ventilation}

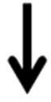

Pre-Processing:

- Missing data imputation

- Check multicollinearity

- Box and Cox transformation

Analysis Cohort
REE ( $n=364)$ repeated
measures

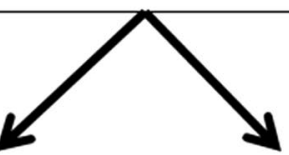

- Center and Scale numeric predictors

- Dummy variables for factors

Feature Engineering

- Derive natural splines

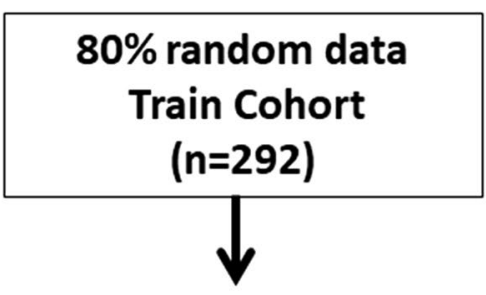

MODEL DEVELOPMENT (TRAIN)

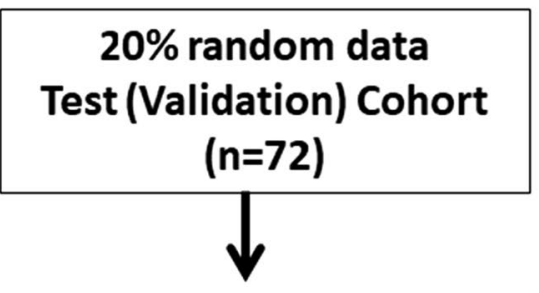

MODEL TESTING (Validation)

\author{
Models optimization \\ hyperparameters using 10 - \\ fold cross-validation \\ (training RMSE)
}

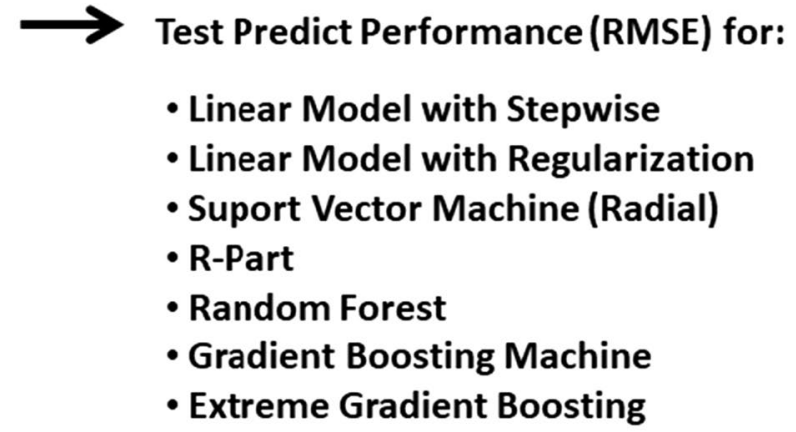

RMSE: root mean square error 
The present study aimed, therefore, to develop a new predictive equation of REE for AKI patients, on dialysis and mechanically ventilated, using a machine learning approach.

\section{Materials and methods}

A cross-sectional descriptive study was carried out in the Dialysis Unit of the Clinical Hospital of Botucatu Medical School. The project was approved by the centre's ethical committee and the protocols used followed the criteria of the Helsinki Declaration (protocol 4383/2012). For development of the predictive equation, 364 indirect calorimetry (IC) measurements in 114 AKI patients on dialysis were consecutively selected, aged between 19 and 95 years. Subjects with a body mass index (BMI) between $18-55 \mathrm{~kg} / \mathrm{m}^{2}$, who gave their consent to participate in the study and agreed to comply with its standards were included. Inclusion criteria were patients admitted to the ICU with a diagnosis of AKI according to the KDIGO criteria [17], clinical symptoms suggestive of sepsis and acute tubular necrosis (ATN), a need for renal replacement therapy (RRT) (stage 3) and mechanically ventilated. Exclusion criteria were patients with AKI of other aetiologies, renal transplanted or those with chronic renal disease stages 4 and 5 (glomerular filtration rate $($ GFR $)<30 \mathrm{ml} / \mathrm{min}$ estimated by the Modification of Diet in Renal Disease (MDRD) equation) [18], a fraction of inspired oxygen $\left(\mathrm{FiO}_{2}\right)$ greater than 0.60 , expiratory positive airway pressure (PEEP) $>10 \mathrm{~cm} \mathrm{H}_{2} \mathrm{O}$, maximum airway pressure $>60 \mathrm{~cm} \mathrm{H}_{2} \mathrm{O}$, stirring presence, use of neuromuscular blockers, air leakage into the ventilator circuit around the endotracheal tube cuff or from a bronchopleural fistula, as these factors lead to inaccuracies in REE measurement by IC. IC was performed using the RMR Quark apparatus (Cosmed, Rome, Italy).

\section{Data analysis}

For construction of the predictive model, $80 \%$ of the cases were randomly separated. Twenty per cent of unused cases were separated for validation. The pre-processing was applied to the training and test set. The continuous predictor variables were transformed using Box and Cox and after dividing by means (centre) and standard deviation (scale). This transformation led to a uniform scale $($ mean $=0, S D=1)$ for all analyses so that they were comparable between analytical platforms. The missing variables were imputed by the median. Categorical variables were converted into dummy variables. In feature engineering, we tested the distribution of each continuous variable according to the outcome. We choose natural splines with three degrees of freedom for age and BMI aims to account for the non-linearity. Afterward, linear and non-linear models were tested in the training

\section{Table 1 Clinical characteristics of the population with AKI on dialysis}

\begin{tabular}{|c|c|}
\hline \multicolumn{2}{|l|}{ Patients parameters $(n=114)$} \\
\hline Age (years) & $60.6 \pm 16.9$ \\
\hline Male gender (\%) & $78(68.4)$ \\
\hline \multicolumn{2}{|l|}{ AKI (\%) } \\
\hline Sepsis-related & $93(81.6)$ \\
\hline ischemic & $13(11.4)$ \\
\hline Nephrotoxic & $6(5.3)$ \\
\hline Mixed & $2(1.8)$ \\
\hline ATN-ISS & $0.64 \pm 0.18$ \\
\hline \multicolumn{2}{|l|}{ Race (\%) } \\
\hline White & $98(86)$ \\
\hline Black & $11(9.6)$ \\
\hline Weight (kg) & $77.6 \pm 22.4$ \\
\hline \multicolumn{2}{|l|}{ Main diagnosis } \\
\hline CVD & $35(30.7)$ \\
\hline Sepsis, severe sepsis, shock & $56(49.1)$ \\
\hline Neoplasia & $9(7.9)$ \\
\hline Liver diseases & $8(7)$ \\
\hline Trauma & $6(5.3)$ \\
\hline Death & $73(63)$ \\
\hline \multicolumn{2}{|c|}{ Patients and IC parameters $(n=364)$} \\
\hline REE using HB (Kcal) & $1540 \pm 346$ \\
\hline REE using IC (Kcal) & $2081 \pm 645$ \\
\hline REE using IC (Kcal/kg/d) & $27.9 \pm 10.4$ \\
\hline \multirow[t]{2}{*}{$\operatorname{VAD}(\mathrm{mcg} / \mathrm{kg} / \mathrm{min})$} & 0.11 \\
\hline & $(0.00-0.35)$ \\
\hline MV & $8.5 \pm 2.8$ \\
\hline Freq. (resp/min) & $17 \pm 5.2$ \\
\hline $\operatorname{PEEP}\left(\mathrm{cm} \mathrm{H}_{2} \mathrm{O}\right)$ & $6 \pm 1.5$ \\
\hline $\mathrm{FIO}_{2}(\%)$ & $38 \pm 10.9$ \\
\hline Temperature $\left({ }^{\circ} \mathrm{C}\right)$ & $37.6 \pm 0.8$ \\
\hline Urea $(\mathrm{mg} / \mathrm{dl})$ & $157 \pm 73$ \\
\hline WBC $\left(\mathrm{mm}^{3}\right)$ & $17,300(12,300-25,500)$ \\
\hline $\mathrm{PCR}(\mathrm{mg} / \mathrm{dl})$ & $23.1(7.6-32.8)$ \\
\hline
\end{tabular}

CVD cardiovascular disease, AKI acute kidney injury, ATN-ISS individual severity score in acute tubular necrosis, REE resting energy expenditure, VAD vasoactive drug, $\mathrm{FIO}_{2}$ fraction of inspired oxygen, $M v$ minute volume, $R R$ respiratory rate, $P E E P$ expiratory positive airway pressure, Creat serum creatinine, WBC white blood-cell count, CRP C-reactive protein

data. These models were: linear regression with stepwise selection, linear regression with regularisation (glmnet), rpart, support vector machine with radial kernel (SVM), generalised boosting machine (GBM), extreme gradient boosting (XGBoost) and random forest. The optimisation of hyperparameters was done in the training set with ten-fold cross-validation and the performance was evaluated by the root mean square error (RMSE). Therefore, we evaluated the final performance of the best model in the test set by choosing the model with the lower RMSE 

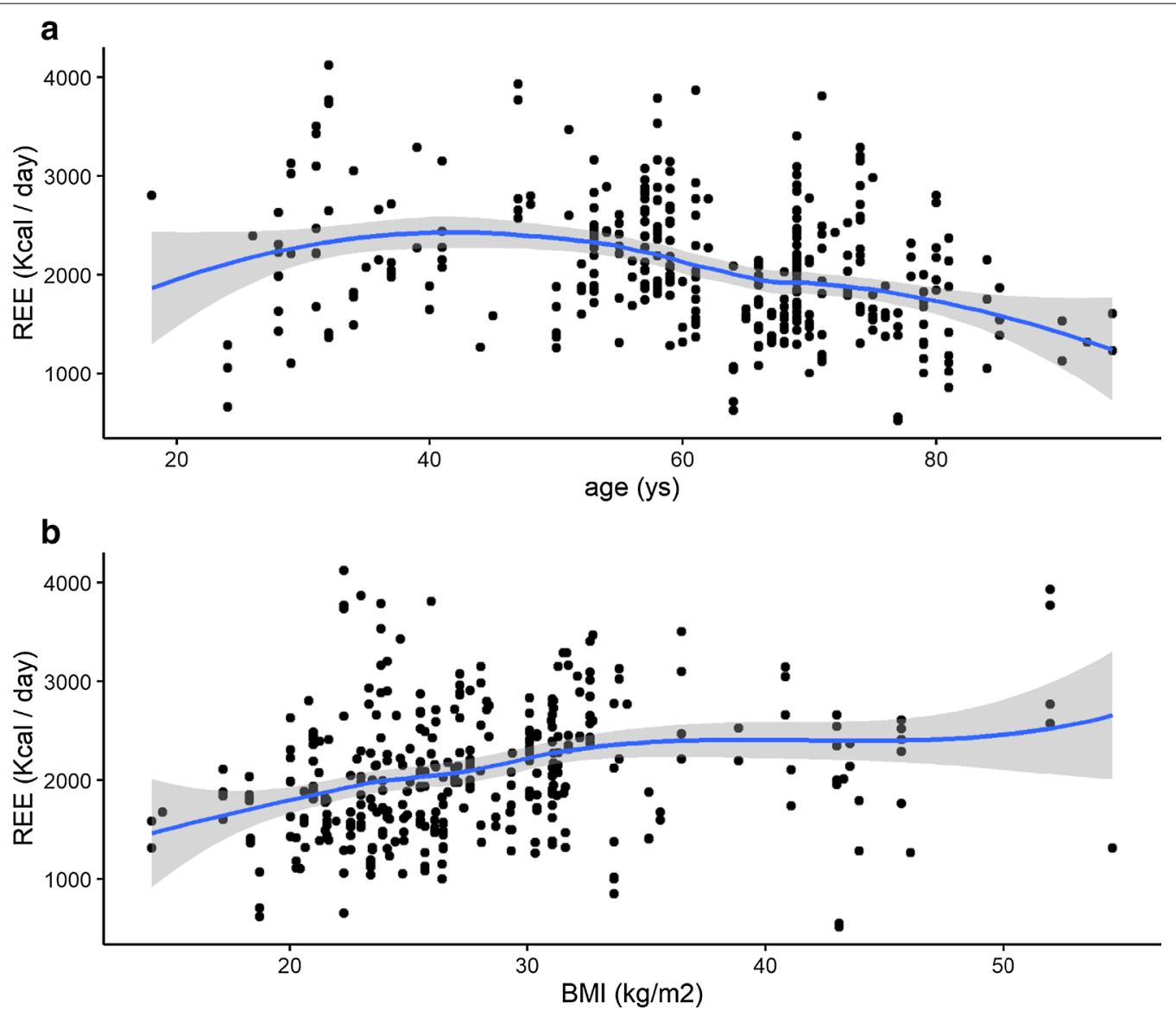

Fig. 2 A plot of age-related to REE (a) and BMI related to REE (b). The blue line is a smooth line that was fitted using polymonial regression

(Fig. 1). The analysis was performed with $\mathrm{R}$ version 3.6.3, Vienna Austria, 2020 with caret package.

\section{Results}

A total of 364 IC measures in 114 patients were evaluated. The mean age was $60.65 \pm 16.9$ years and $68.4 \%$ were males. Diagnoses of sepsis and cardiovascular disease (CVD) accounted for $79.8 \%$ of hospitalisations. AKI was the aetiology associated with sepsis in most patients $(81.6 \%)$. The REE estimated by the Harris-Benedict $(\mathrm{HB})$ formula averaged $1540 \pm 346 \mathrm{kcal}$; whereas the REE measured by the IC was significantly higher $2081 \pm 645 \mathrm{kcal}(27.7 \pm 10 \mathrm{kcal} / \mathrm{kg} /$ day, $p<0.001)$. Table 1 shows the clinical characteristics of the general population studied at the time when dialysis was indicated.

The variables available to train the models of REE in the total population $(n=364)$ are summarised in attachment 01 . In the exploratory data analysis, we plotted the REE related to age and BMI (Fig. 2). We demonstrated a non-linear association between these predictors and the outcome. Then, we applied natural splines with three degrees of freedom aiming to capture the non-linearity (feature engineering).

We trained different algorithms by ten-fold cross-validation in the training set ( $80 \%$ of the data) and the hyperparameters of each model were tuned based in the lower RMSE. After training tuned models, we applied resamples of the training data and selected the best model based on the lower RMSE (Fig. 3). Therefore, the final models were evaluated for performance in the test set (internal validation) aiming to confirm the results of the training set (Table 2).

The models that had a non-linear approach had a lower RMSE. The linear model may have had the advantage of simplicity and interpretability (attachment 02). The model with the best accuracy was selected by the lower RMSE: random forest. The variable importance of the final model (random forest) was plotted in Fig. 4.

Finally, we plotted a correlation between the predicted values of REE and observed REE in the test set (unseen data). The random forest model (best model) had a final 

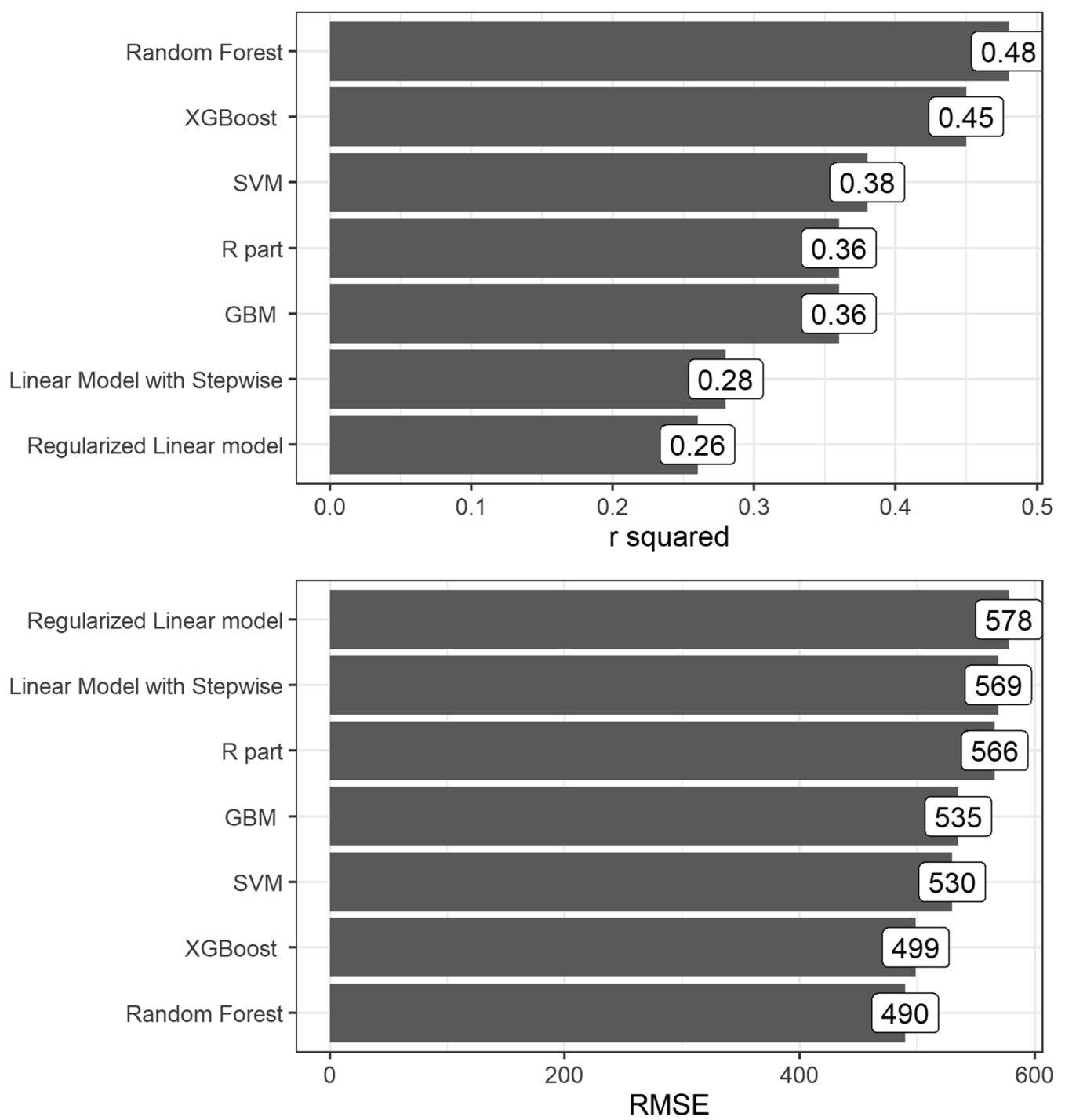

Fig. 3 Summary statistics of tuned trained models in resamples of the training set. The box summaries the mean values. The statistics were: $R$ squared and root mean square error (RMSE)

r-value in this test set of 0.69 compared to an r-value of 0.24 with the Harris-Benedict equation (Fig. 5).

\section{Discussion}

Information about the energy expenditure assessment of patients with AKI is scarce in the literature. Our study found that the REE estimated by the Harris and Benedict formula [19] was significantly lower than that measured by IC. This finding corroborates the indication not to use this formula in critically ill patients and in patients with AKI $[11,14,20,21]$ and the need to propose a new equation for those AKI on dialysis [21-25].

It has long been held that critical illness is a hypermetabolic state, i.e. that the basal metabolic rate is higher than that predicted by simple population characteristics
[25-27]. Physiological factors such as fever, increased substrate cycling, and synthetic functions associated with the host response to stress and inflammation provide a

Table 2 Summary statistics of final tuned models in the test set (20\% of the data). $R^{2}$ ( $R$ squared), and root mean square error (RMSE)

\begin{tabular}{lll}
\hline Models & $\mathbf{R}^{\mathbf{2}}$ & RMSE \\
\hline Linear model with stepwise & 0.28 & 569.24 \\
Regularized linear model & 0.26 & 578.44 \\
SVM & 0.38 & 530.05 \\
Random forest & 0.48 & 490.05 \\
R part & 0.36 & 566.61 \\
GBM & 0.36 & 535.88 \\
XGBoost & 0.45 & 499.75 \\
\hline
\end{tabular}


theoretical basis for this generalization. Energy expenditure is also influenced by common ICU therapies [14,25].

This study aimed to develop and validate predictive equations for REE in severe AKI patients using a machine learning approach. It was found that the models were developed validly and significantly predicted REE in these patients and according to several linear and non-linear algorithms. In the present study, REE was positively correlated with C-reactive protein, minute volume (MV), expiratory positive airway pressure, serum urea, BMI and inversely with age (attachment 02 ). The principal variables included in the best model were age, BMI, use of vasopressors, expiratory positive airway pressure, minute volume, C-reactive protein, temperature and serum urea. The final $r$-value in the validation set was 0.69 . In the literature, there is no consensus regarding the procedures to be used for the validation of predictive models. Some authors do not suggest the use of determination and correlation coefficients for the validation of techniques or estimated variables [15]. Others consider that the Bland Altman plot is likely to show a systematic proportion bias [19]. We used ten-fold cross-validation and selected the model with the lower RMSE. The performance of the best model was confirmed in a test set of $20 \%$ randomly selected unseen data (internal validation). The linear models had the advantage of simplicity of the model built and better interpretability but did not capture the non-linearity of the data. We confirmed this with a very low accuracy of the Harris-Benedict equation in the test set. The use of natural splines to age and BMI predictors improved the linear models but did not reach the same performance as the non-linear models like boost trees or support vector machines [22, 23]. The best model had a higher performance but a trade-off in lower interpretability. In addition, the traditional model like the Harris-Benedict [19] uses linear models that were easy to implement. Otherwise, the implementation of a model, like the random forest, that requires a calculator. However, currently, we have the facility of computers or apps that may overcome this difficulty.

Another advantage of the machine learning approach is to demonstrate other predictor variables that influence the outcome by finding complex interactions. The principal factors that contributed to the variability of REE were the BMI and age that were previously described with traditional models $[24,25]$. The machine analysis in this study reveals other new contributary variables that predict the REE, for example, ventilator parameters and biochemical values. Otherwise, a simple linear model with this data set without pre-processing and feature engineering results in a model with lower accuracy and does not shown new interactions $\left(R^{2}=0.21\right.$, data not shown $)$.

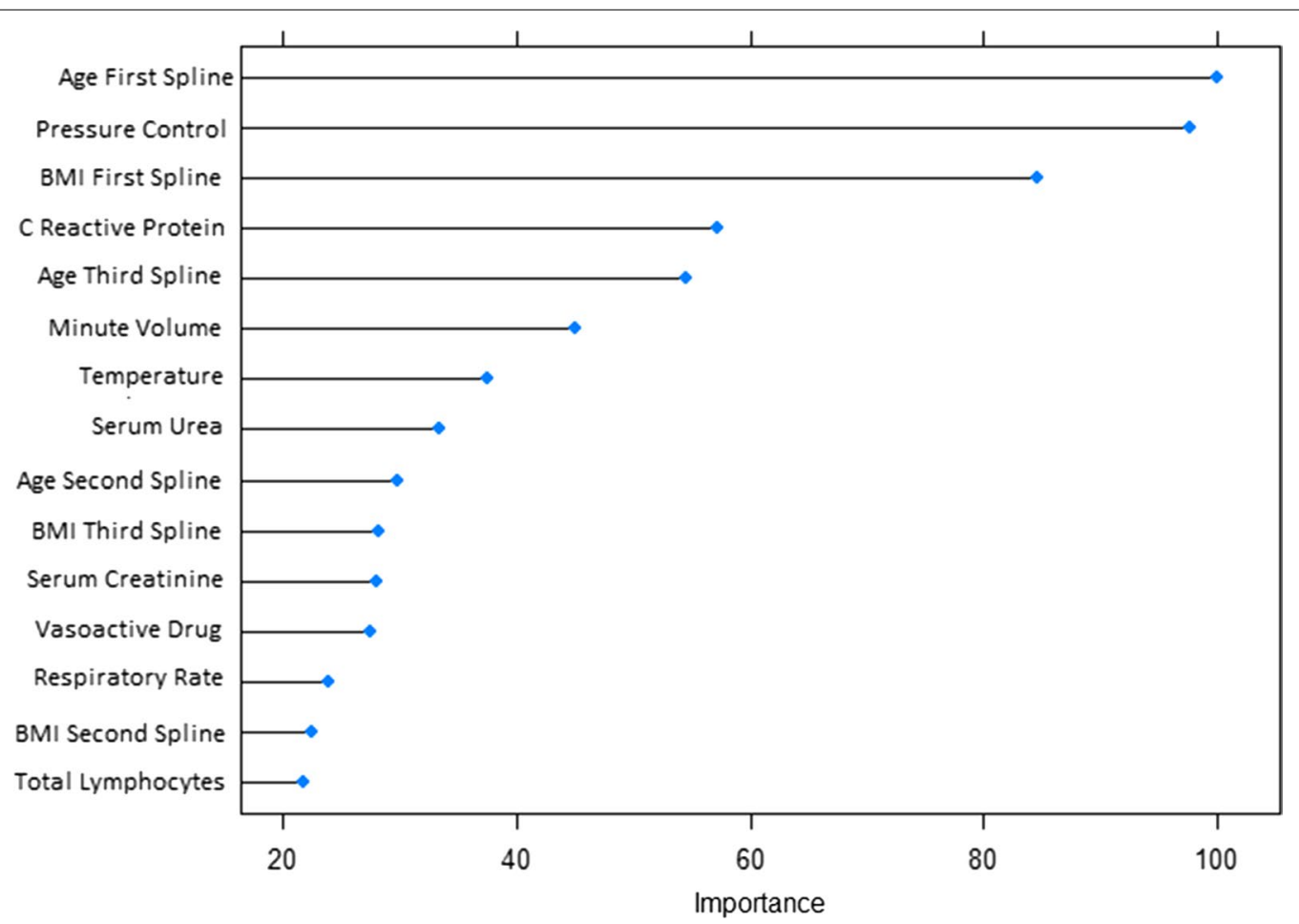

Fig. 4 Variable importance in the random forest model. The importance was reported on a normalised scale 

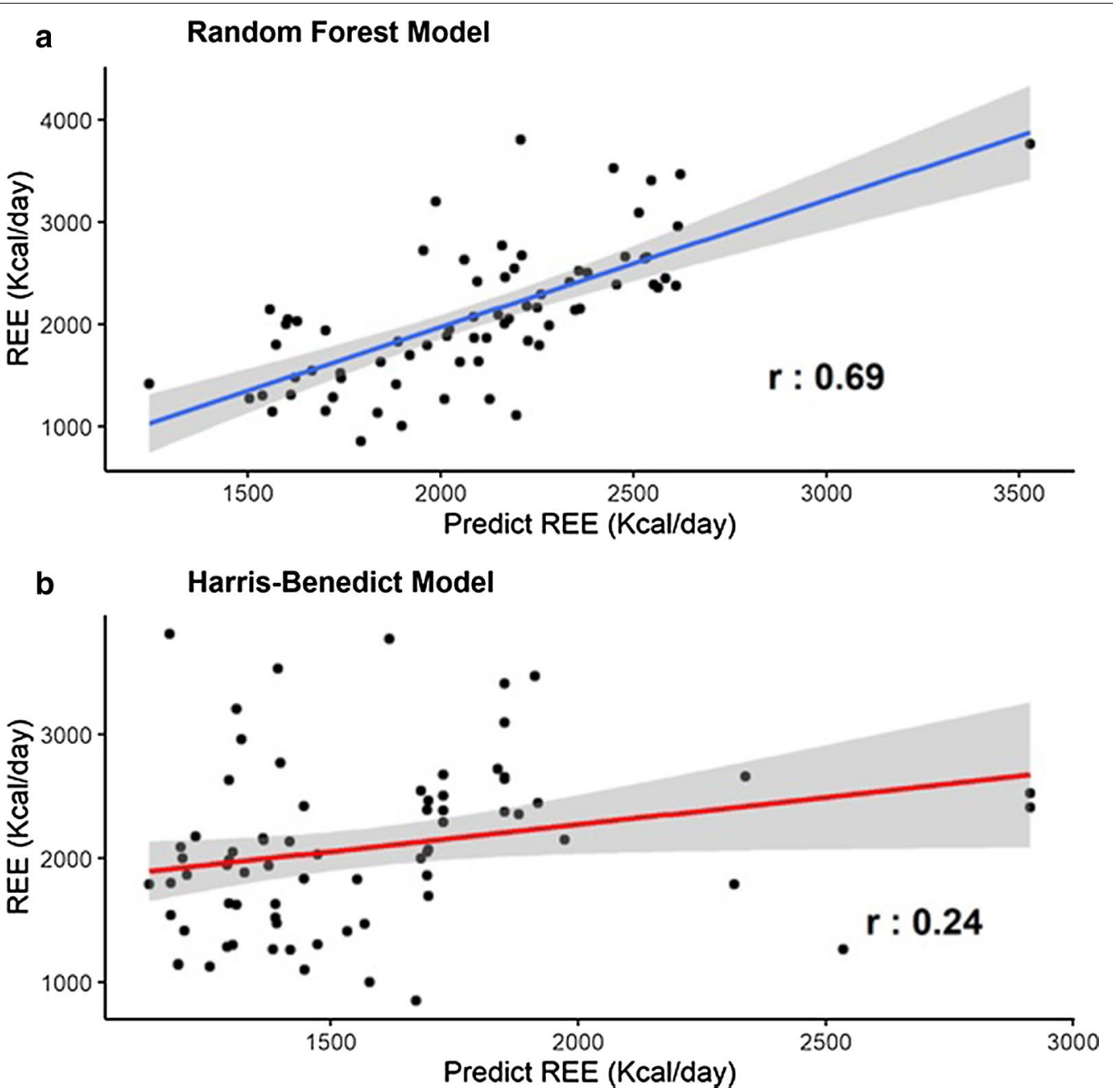

Fig. 5 A correlation plot between the predicted and observed REE in the test set (20\% of unseen data). a Predicted REE by random forest model and $\mathbf{b}$ predicted REE by Harris-Benedict equation. We had an r-value of 0.69 with the random forest model (new model) and an r-value of 0.24 with the Harris-Benedict

Thus, this study demonstrates the importance of estimating the REE in severe AKI patients, which is a determining factor in the assessment of nutrition status in critically ill patients. Previous studies reported correlations between morbidity or mortality and REE and indicated that sicker patients and non-survivors had a lower REE $[22,25]$ but this is not a universal observation [26, 27]. Interpretations of these findings are limited by potential survivorship bias and confounders such as age and physical activity.

From a practical and application point of view, it is worth mentioning the capacity for technical evaluations to reduce the difference in REE between IC and that estimated by other conventional formulas, such as $\mathrm{HB}$, the use of these predictive equations for the assessment of passive and active trawling, contributing with relevant information for nutritionists and physicians.
The present study has as main limitations the fact that the predictive models are not valid for non- severe AKI patients and the Bland Altman model was not performed. Although we trained models with a robust estimator with ten-fold cross-validation using RMSE as a metric, an approach regularly used in machine learning analysis, we tested the model in unseen data that may be considered an internal validation.

\section{Conclusion}

We propose a new predictive equation for estimating the REE of AKI patients on dialysis that uses a non-linear approach with better performance than actual models. 


\section{Authors' contributions}

CRG and DP analysed and interpreted the patient data regarding acute kidney injury disease and resting energy. DP and LGMA contributed in writing the manuscript. LGMA performed the statistical analysis. All authors read and approved the final manuscript.

\section{Funding}

The study did not receive funding.

\section{Availability of data and materials}

All data generated or analysed during this study can be included in this published article as supplementary information files.

\section{Ethics approval and consent to participate}

The project was approved by the ethical committee of Botucatu Medical School and the protocols used followed the criteria of the Helsinki Declaration (protocol 4383/2012).

\section{Consent for publication}

Not applicable.

\section{Competing interests}

The authors declare that they have no competing interests.

Received: 29 June 2020 Accepted: 21 October 2020

Published online: 17 November 2020

\section{References}

1. VA/NIH Acute Renal Failure Trial Network, Palevsky PM, Zhang JH, O'Connor TZ, Chertow GM, Crowley ST, et al. Intensity of renal support in critically ill patients with acute kidney injury. N Engl J Med. 2008;3(359):7-20.

2. Hanna J, Nichol A. Acute renal failure and the critically ill. Anaesth Intensive Care Med. 2012;1(13):166-70.

3. Dvir D, Cohen J, Singer P. Computerized energy balance and complications in critically ill patients: an observational study. Clin Nutr. 2006;25:37-44

4. Fiaccadori E, Lombardi M, Leonardi S, Rotelli CF, Tortorella G, Borghetti A. Prevalence and clinical outcome associated with preexisting malnutrition in acute renal failure: a prospective cohort study. J Am Soc Nephrol. 1999;10:581-93.

5. McCarthy MS, Phipps SC. Special nutrition challenges: current approach to acute kidney injury. Nutr Clin Pract. 2014;29:56-62.

6. Berbel MN, Góes RC, Balbi AL, Ponce D. Nutritional parameters are associated with mortality in acute kidney injury. Clinics. 2014;69:476-82.

7. Schlein KM, Coulter SP. Best practices for determining resting energy expenditure in critically ill adults. Nutr Clin Pract. 2014;29:44-55.

8. Haugen HA, Chan L-N, Li F. Indirect calorimetry: a practical guide for clinicians. Nutr Clin Pract. 2007;22(4):377-88.

9. Singer $P$, Singer J. Clinical guide for the use of metabolic carts: indirect calorimetry-no longer the orphan of energy estimation. Nutr Clin Pract. 2016;31:30-8.

10. Faisy C, Guerot E, Diehl JL, Labrousse J, Fagon JY. Assessment of resting energy expenditure in mechanically ventilated patients. Am J Clin Nutr. 2003;78:241-9.

11. Frankenfield D, Hise M, Malone A, Russell M, Gradwell E, Compher C. Prediction of resting metabolic rate in critically ill adult patients: results of a systematic review of the evidence. J Am Diet Assoc. 2007;107:1552-61.

12. Frankenfield DC, Coleman A, Alam S, Cooney RN. Analysis of estimation methods for resting metabolic rate in critically ill adults. JPEN J Parenter Enteral Nutr. 2009:33:27-36.
13. MacDonald A, Hildebrandt L. Comparison of formulaic equations to determine energy expenditure in the critically ill patient. Nutrition. 2003:19:233-9.

14. de Góes CR, Berbel-Bufarah MN, Sanches AC, Xavier PS, Balbi AL, Ponce D. Poor agreement between predictive equations of energy expenditure and measured energy expenditure in critically ill acute kidney injury patients. Ann Nutr Metab. 2016;68:276-84. https://doi.org/10.1159/00044 6708.

15. Costa SD, de Andrade LGM, Barroso FVC, Oliveira CMC, Daher EF, Fernandes PFCBC, et al. The impact of deceased donor maintenance on delayed kidney allograft function: a machine learning analysis. PLoS One. 2020;15(2):0228597. https://doi.org/10.1371/journal.pone.0228597 (eCollection 2020).

16. Armbruster M, Rist M, Seifert S, Frommherz L, Weinert C, Mack C, et al. Metabolite profiles evaluated, according to sex, do not predict resting energy expenditure and lean body mass in healthy non-obese subjects. Eur J Nutr. 2019;58(6):2207-17. https://doi.org/10.1007/s0039 4-018-1767-1.

17. Kidney Disease: Improving Global Outcomes (KDIGO) Acute Kidney Injury Work Group. KDIGO clinical practice guideline for acute kidney injury. Kidney Int Suppl. 2012;2:1-138.

18. Levey AS, Bosch JP, Lewis JB, Greene T, Rogers N, Roth D. A more accurate method to estimate glomerular filtration rate from serum creatinine: a new prediction equation. Modification of Diet in Renal Disease Study Group. Ann Intern Med. 1999;16(130):461-70.

19. Harris JA, Benedict FG. A biometric study of human basal metabolism. Proc Natl Acad Sci USA. 1918:4:370-3.

20. Kross EK, Sena M, Schmidt K, Stapleton RD. A comparison of predictive equations of energy expenditure and measured energy expenditure in critically ill patients. J Crit Care. 2012;27:321. e5-12. https://doi. org/10.1016/j.jcrc.2011.07.084.

21. Hickmann CE, Roeseler J, Castanares-Zapatero D, Herrera El, Mongodin A, Laterre PF. Energy expenditure in the critically ill performing early physical therapy. Intensive Care Med. 2014;40:548-55. https://doi.org/10.1007/ s00134-014-3218-7.

22. Zusman O, Kagan I, Bendavid I, Theilla M, Cohen J, Singer P. Predictive equations versus measured energy expenditure by indirect calorimetry: a retrospective validation. Clin Nutr. 2019;38(3):1206-10. https://doi. org/10.1016/j.clnu.2018.04.020.

23. Weng SF, Reps J, Kai J, Garibaldi JM, Qureshi N. Can machine-learning improve cardiovascular risk prediction using routine clinical data? PLoS ONE. 2017;12(4):e0174944. https://doi.org/10.1371/journal.pone.0174944 (eCollection 2017).

24. Astrup A, Buemann B, Christensen NJ, Madsen J, Gluud C, Bennett P, Svenstrup B. The contribution of body composition, substrates, and hormones to the variability in energy expenditure and substrate utilization in premenopausal women. J Clin Endocrinol Metab. 1992;74(2):279-86 (J Clin Epidemiol. 2020. https://doi.org/10.1016/j.jclinepi.2020.03.005).

25. Góes CR, Balbi AL, Ponce D. Evaluation of factors associated with hypermetabolism and hypometabolism in critically ill AKI patients. Nutrients. 2018. https://doi.org/10.3390/nu10040505.

26. Zusman O, Theilla M, Cohen J, Kagan I, Bendavid I, Singer P. Resting energy expenditure, calorie and protein consumption in critically ill patients: a retrospective cohort study. Crit Care. 2016;20(1):367. https:// doi.org/10.1186/s13054-016-1538-4.

27. Wu C, Wang X, Yu W, et al. Hypermetabolism in the initial phase of intensive care is related to a poor outcome in severe sepsis patients. Ann Nutr Metab. 2015;66(4):188-95. https://doi.org/10.1159/000430848.

\section{Publisher's Note}

Springer Nature remains neutral with regard to jurisdictional claims in published maps and institutional affiliations. 